\section{FORTRAN IV functions for computing expected half-normal scores}

\section{WILLIAM P. DUNLAP and LEANN MYERS \\ Tulane University, New Orleans, Louisiana}

A half-normal distribution results if all those values that are negative in a standard normal distribution are made positive; that is, it is the distribution of the absolute values of normally distributed data. The mathematical formula for this distribution is

$$
(2 / \mathrm{PI})^{1 / 2} \operatorname{EXP}\left(-Z^{2} / 2\right)
$$

for $\mathrm{Z}$ greater than or equal to zero. If one were to sample $\mathrm{N}$ values from the half-normal distribution and put those scores in order, the smallest value would be near zero and the largest value in the neighborhood of 2 to 3 . The program described in this paper computes the expected value of the $k$ th of $N$ ordered half-normal scores.

Although there are a number of potential applications of expected half-normal scores, only three will be described in detail. Hills (1969) and Stavig and Acock (1976) proposed a test for the significance of correlation coefficients in a matrix, in which there may be a great number of coefficients to be tested. In such a case, there should be several rather large coefficients by chance alone. Their procedure is to rank the correlations in terms of absolute value, convert the ordered corre. lations to normal scores by Fisher's $\mathrm{z}$ transformation, and then compare these with the expected values of halfnormal scores. Only those correlations whose z-score equivalents surpass the corresponding expected ordered half-normal score by a sufficient amount are considered significant.

Daniel (1959) and Zahn (1975a, 1975b) described the use of expected half-normal scores for interpreting the results of factorial experiments in which all the independent variables have only two levels. The idea here is that if one runs a five-way factorial, 31 tests of significance would be made, some of which would appear to be quite large by chance alone. Since each effect is on a single degree of freedom, each can be considered as a linear contrast of the cell means in the experiment. These contrasts should generally have normal distributions, so that if ranked, they may be compared with corresponding expected half-normal scores, as were the transformed correlations above.

Third, an easy way to compare the absolute values of quantities that should be normal with the expected values of ordered half-normal scores is to construct a

The authors' mailing address is: Department of Psychology, Tulane University, New Orleans, LA 70118. half-normal probability plot. Such plots are produced by programs such as BMDP5D, from the BMDP series of programs (Dixon, 1981). Essential to such plots is the computer generation of expected half-normal scores. Other applications for these scores exist: Best, Cameron, and Eagleson (1983) described the use of half-normal probability plots for testing large sets of values of Kendall's Tau; Cox and Lauh (1967) described the use of half-normal probability plots in analyzing multidimensional contingency tables; and Stavig (1978) provided transformational equations that permit the application of half-normal score techniques to $t, F$, and $\chi^{2}$ statistics.

The critical problems for all of these potential applications are the lack of available tables of expected halfnormal scores and the lack of accurate algorithms to approximate these scores. The only table we were able to find is in Zahn (1975b), which goes up to $\mathrm{N}=20$, clearly insufficient for many of the applications previously described. Although several approximating algorithms have been suggested, the accuracy of most currently available algorithms is poor. Therefore, the purposes of the present paper are to provide a computer program that accomplishes an exact solution via numerical integration, to assess the relative merits of approximating algorithms, and to provide a quickly executing function for estimating expected half-normal scores with reasonable accuracy.

Table 1 compares the accuracy of each approximation with the exact solution; the approximations are ordered in terms of poorest to best accuracy. The first two use the inverse of the normal probability integral, for which we used the approximating function of Emerson (1979). The function labeled S\&A-1 was proposed by Stavig and Acock (1976) to be used when tables of expected normal scores were not available. The function is

$$
\mathrm{z}^{-1}(1-(\mathrm{J}-1 / 2) / 2 \mathrm{~N})
$$

where $\mathrm{N}$ is the number of half-normal scores and $\mathrm{J}$ is the position of the score in order. The second function is that used by the program BMDP5D in making halfnormal probability plots (Dixon, 1981); the function is

$$
\mathrm{z}^{-1}[(3 \mathrm{~N}+3 \mathrm{~J}-1) /(6 \mathrm{~N}+1)] \text {. }
$$

It appears that both of these functions are inaccurate to an extent that one should question using them in halfnormal plots, much less in hypothesis testing. The remaining three functions tested are based upon the use of expected ordered normal scores to approximate expected ordered half-normal scores; the former are computed quickly via an algorithm described by Dunlap and Brown (1983). The function labeled S\&A-2, also described by Stavig and Acock (1976), is 
Table 1

Absolute Error of Five Algorithms Approximating Ordered Half-Normal Scores as Functions of Number of Scores

\begin{tabular}{llccccccc}
\hline & & \multicolumn{7}{c}{ Number of Half-Normal Scores } \\
\cline { 3 - 8 } Algorithm & & 2 & 4 & 8 & 16 & 32 & 64 & 128 \\
\hline \multirow{2}{*}{ S\&A-1 } & 1st & .022 & .070 & .080 & .077 & .071 & .064 & .0584 \\
& Max & .149 & .105 & .080 & .077 & .071 & .064 & .0584 \\
BMDP81 & 1st & .108 & .059 & .042 & .036 & .034 & .033 & .0330 \\
& Max & .174 & .11 & .065 & .036 & .034 & .033 & .0330 \\
S\&A-2 & 1st & .099 & .041 & .017 & .007 & .002 & .001 & .0002 \\
& Max & .171 & .110 & .064 & .019 & .019 & .009 & .0048 \\
A1t-1 & 1st & .034 & .020 & .011 & .006 & .004 & .002 & .0010 \\
& Max & .034 & .027 & .011 & .006 & .004 & .002 & .0010 \\
A1t-2 & 1st & .000 & .000 & .000 & .000 & .000 & .000 & .0002 \\
& Max & .007 & .008 & .007 & .004 & .003 & .002 & .0012 \\
\hline
\end{tabular}

Note-Rows labeled 1st contain errors for the largest score, and Max, the largest difference between estimated and actual scores.

$\operatorname{ENS}(\mathrm{K}, \mathrm{M}) ; \mathrm{M}=2 \mathrm{~N}, \mathrm{~K}=1$ to $\mathrm{N}$,

where ENS represents expected ordered normal scores. Somewhat better overall accuracy can be obtained by using a slightly altered function.

$$
\operatorname{ENS}(\mathrm{K}, \mathrm{M}) ; \mathrm{M}=2 \mathrm{~N}+1, \mathrm{~K}=1 \text { to } \mathrm{N} \text {. }
$$

This function is labeled A1t-1 in Table 1; however, it consistently produces slight overestimates of the correct values. The final function, labeled Alt-2, is the same as Alt-1 except that an error correction based on $\mathrm{N}$ is subtracted to improve the estimate. The error correction $(\mathrm{EC})$ is

$$
\mathrm{EC}=.092263 \mathrm{~N}^{-1}-.057445 \mathrm{~N}^{-2}+.012751 \mathrm{~N}^{-3}+.000443 .
$$

The FORTRAN IV code for Alt-2 appears in Appen$\operatorname{dix} A$; it is quite accurate for the large half-normal scores, which are most crucial to hypothesis testing, and is accurate to at least two decimal places for all other scores.

The exact solution for expected ordered half-normal scores via numerical integration is quite parallel to a similar exact solution for expected normal scores described by Dunlap and Brown (1983). The half-normal probability function is calculated from the normal probability function using an approximation (number 26.2.11) from Zelen and Severo (1965). Simpson's rule is used to numerically integrate the function.

$$
\frac{\mathrm{N} !}{(\mathrm{N}-\mathrm{J}) !(\mathrm{J}-1) !} \mathrm{P}^{(\mathrm{N}-\mathrm{J})} \mathrm{Q}^{(\mathrm{J}-1)} \mathrm{zdx}
$$

from zero to positive infinity, where $\mathrm{P}$ is the proportion below $\mathrm{x}$ and $\mathrm{Q}$ the proportion above $\mathrm{x}$ on the halfnormal distribution, and $\mathrm{z}$ is the ordinate of the halfnormal curve at $x$. First, the program searches for a usable range for the integration; then Simpson's rule is applied over 101 steps. The values computed agree exactly with the three-decimal place values tabled by Zahn (1975b); however, the present program is probably accurate to five decimal places because it uses the same computational scheme that was used for expected normal scores by Dunlap and Brown (1983), which was accurate to that level. The FORTRAN code for the exact solution is available in Appendix B.

Time Requirements. The shortcut approximate function computes a single expected half-normal score in approximately $0.28 \mathrm{msec}$ on the DEC-20 computer. Computation times for the exact solution are considerably longer and depend on both $\mathrm{N}$ and $\mathrm{J}$. The shortest computing times, of approximately $175 \mathrm{msec}$, were found when the smallest score was computed and the $\mathrm{N}$ was approximately 90 . The longest computing times were found when computing the largest halfnormal scores; these increased as a function of $\mathrm{N}$, taking as long as $590 \mathrm{msec}$ at $\mathrm{N}=2,000$.

Program Availability. These functions are written in standard FORTRAN IV and run on a DEC-20 computer. Main routines are included before each function for instructive purposes. These main routines contain some DEC-specific FORTRAN statements; users will probably wish to write their own main programs, depending upon the use they have in mind. Neither function uses dimensioned storage; therefore, memory size need only be sufficient to load and run the programs. Listings of these functions can be obtained at no cost from William P. Dunlap, Department of Psychology, Tulane University, New Orleans, Louisiana 70118.

\section{REFERENCES}

Best, D. J., Cameron, M. A., \& Eagleson, G. K. (1983). A test for comparing large sets of tau values. Biometrika, 70, 447-453.

Cox, D. R., \& LAUH, E. (1967). A note on the graphical analysis of multidimensional contingency tables. Technometrics, 9, $481-488$.

DANIEL, C. (1959). Use of half-normal plots in interpreting factorial two-level experiments. Technometrics, 1, 311-341. 
Dixon, W. J. (1981). BMDP statistical software. Berkeley: University of California Press.

DunlaP, W. P., \& Brown, S. G. (1983). FORTRAN IV functions to compute expected normal scores. Behavior Research Methods \& Instrumentation, 15, 395-397.

Emerson, P. L. (1979). Computer approximation of the inverse of the normal distribution function. Behavior Research Methods \& Instrumentation. 11, 397-398.

Hills, M. (1969). On looking at large correlation matrices. Biometrika, 56, 249-253.

Stavig, G. R. (1978). Applying multiple comparison techniques to $\mathrm{z}, \mathrm{t}, \chi^{2}$, and $\mathrm{F}$ based statistics. Political Methodology, 5, 199-212.

Stavig, G. R., \& Acock, A. C. (1976). Evaluating the degree of dependence for a set of correlations. Psychological Bulletin, 83, 236-241.

ZAHN, D. A. (1975a). An empirical study of the half-normal plot. Technometrics, 17, 201-211.

ZAHN, D. A. (1975b). Modifications of and revised critical values for the half-normal plot. Technometrics, 17, 189-200.

Zelen, M., \& Severo, N. C. (1965). Probability functions. In M. Abramowitz \& I. A. Stegun (Eds.), Handbook of mathematical functions. New York: Dover.

\section{Appendix A}

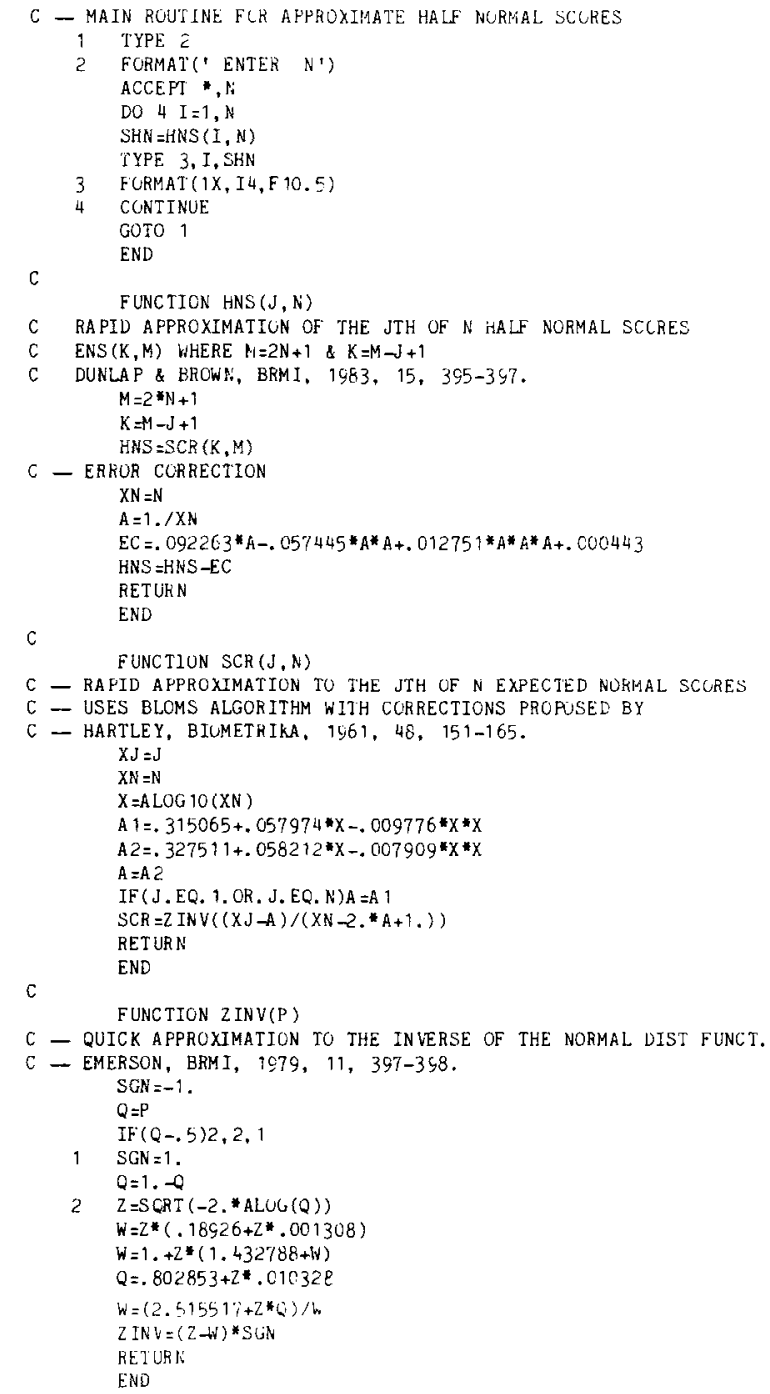


$X=D A B S(X X)$

POF $2=1$. DO

IF (X.GT. . . 35)GO10 3

$S=X$

$\mathrm{T}=\mathrm{X}$
$\mathrm{C}=\mathrm{X} * \mathrm{X}$

$X N=0.00$

$1 \quad X N=X N+1 . D O$

$T=T * C /(2, D O * X N+1, D O)$

$\mathrm{SN}=\mathrm{S}+\mathrm{T}$

IF (SN.EQ.S)GOTO 2
$S=S N$

GOTO 1

POF Z $=.5 D 0+.3989422804014327 D 0 * D E X P(-C / 2 . D O)=S$

3 IF (XX.LT. O. DO)POF Z $=1$. DO-POEZ

RETUR

END

(Revision accepted for publication June 5, 1984.) 